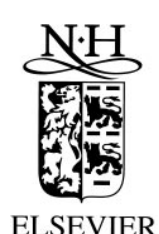

\title{
Conductance histograms and conducting channels in atomic-scale metallic contacts
}

\author{
T. López-Ciudad ${ }^{1}$, A. García-Martín, A.J. Caamaño, J.J. Sáenz * \\ Departamento de Física de la Materia Condensada and Instituto de Ciencia de Materiales 'Nicolás Cabrera', \\ Universidad Autónoma de Madrid, E-28049 Madrid, Spain
}

Received 9 March 1999; accepted for publication 5 August 1999

\begin{abstract}
Properties of the conductance and transport channels of atomic-scale contacts are discussed within the free electron model. In agreement with recent experiments, we find that, owing to the contribution of evanescent channels, even when the conductance is close to an integer number $N$ of conductance quanta more than $N$ channels may contribute significantly to the current. We show that the observation of peaks at integer multiples of $G_{0}$ in conductance histograms is not a signature of conductance quantization of individual contacts. However, the observed peaks can still be associated to the quantum nature of electron transport in metallic contacts. (C) 1999 Elsevier Science B.V. All rights reserved.
\end{abstract}

Keywords: Contacts; Electrical transport (conductivity, resistivity, mobility etc.); Jellium models; Quantum effects; Semi-empirical models and model calculations

\section{Introduction}

The study of the conductance of metallic contacts of atomic dimensions have been the object of great interest in the last few years [1,2]. According to the scattering approach to electric transport, the conductance can be described in terms of a transmission matrix $\mathrm{t}$, and is given by the Landauer-Büttiker formula, $G=G_{0} \operatorname{tr}\left(t t^{\dagger}\right)=G_{0} \sum_{i} T_{i}$ where $G_{0}=2 e^{2} / h$ is the quantum of conductance and $T_{i}$ are the eigenvalues of $t t^{\dagger}$. (i.e. transport eigenchannels [3,4]).

\footnotetext{
* Corresponding author. Fax: +34 913973961 .

E-mail address: juanjo.saenz@uam.es (J. Sáenz)

${ }^{1}$ Present address: Instituto de Ciencia de Materiales, CSIC, Campus de Cantoblanco, 28049 Madrid, Spain.
}

When $T_{i} \approx 1, G$ is simply given by $G_{0} N$, where $N$ is the number of propagating channels, and the conductance is then quantized.

In metallic contacts, the behavior of the conductance with elongation is a stepwise curve. In general, the position of the steps changes from one experiment to another. However, for a wide range of metals, experimental conductance histograms obtained from a large series of runs of contact breaking [1,2,5-8] demonstrate unambiguously the existence of clear peaks at integer numbers of $2 e^{2} / h$. Recent experimental results [9] have shown that, even when the total conductance is close to an integer number $N$ of conductance quanta, more than $N$ channels contribute significantly to the current. For single atom contacts, the experiments 
together with 'tight-binding' calculations [10], show a direct link between valence orbitals and the number of conduction channels. As we will see, this relationship can be understood within a free electron model in contrast with earlier suggestions [10].

These results raised intriguing questions about the nature of the observed conductance quantization: "What makes atomic-size contacts adopt, statistically, configurations with nearly integer values of the conductance?" [9]; "Do conductance histograms constitute a proof for conductance quantization?" [8] ; "Do histogram peaks reflect general statistical properties of quantum transport [11] or are they the result of favorable atomic configurations [8]?". The aim of this Letter is to provide theoretical insight into these questions within a free electron approach.

We will first focus on the conductance of singleatom contacts. According to the free electron model [12-14], the main signature of chemical valence $Z$ in the electrical conduction through metallic contacts would be the ratio between the atomic radius $R_{0}$ (the Wigner-Seitz radius) and the Fermi wavelength $\lambda_{\mathrm{F}}$ :

$R_{0} / \lambda_{\mathrm{F}}=0.305 Z^{1 / 3}$.

The properties of the electric transport depend, not only on $R_{0} / \lambda_{\mathrm{F}}$ but also on the local geometry of the contact which can be characterized by the effective opening angle $\theta$ [15] (see Fig. 1a). The experimental shapes of gold contacts obtained in a high resolution transmission electron microscope (HRTEM) [16,17] show typical opening angles of the order of $\sim 45^{\circ} 60^{\circ}$. In some cases, instead of a single atom contact, an atomic wire made of a row of suspended atoms between the two electrodes has been observed $[17,18]$. The effective angle $\theta$ should then be close to zero. In Fig. 1b we have plotted the conductance versus contact cross-section $A / \lambda_{\mathrm{F}}^{2}$ obtained for hyperbolic constrictions with hard-wall boundary conditions for two different opening angles (see Fig. 1a) $\theta=0$ and $60^{\circ}$ [15] . The corresponding eigenchannel decomposition for $\theta=60^{\circ}$ is shown in Fig. 1c. A contact cross-section made of a single atom would have, in the free electron approach, an effective area $A / \lambda_{\mathrm{F}}^{2} \approx \pi\left[\left(R_{0}+\delta R\right) / \lambda_{\mathrm{F}}\right]^{2}$ where $\delta R / \lambda_{\mathrm{F}}$ is a 'soft-wall'
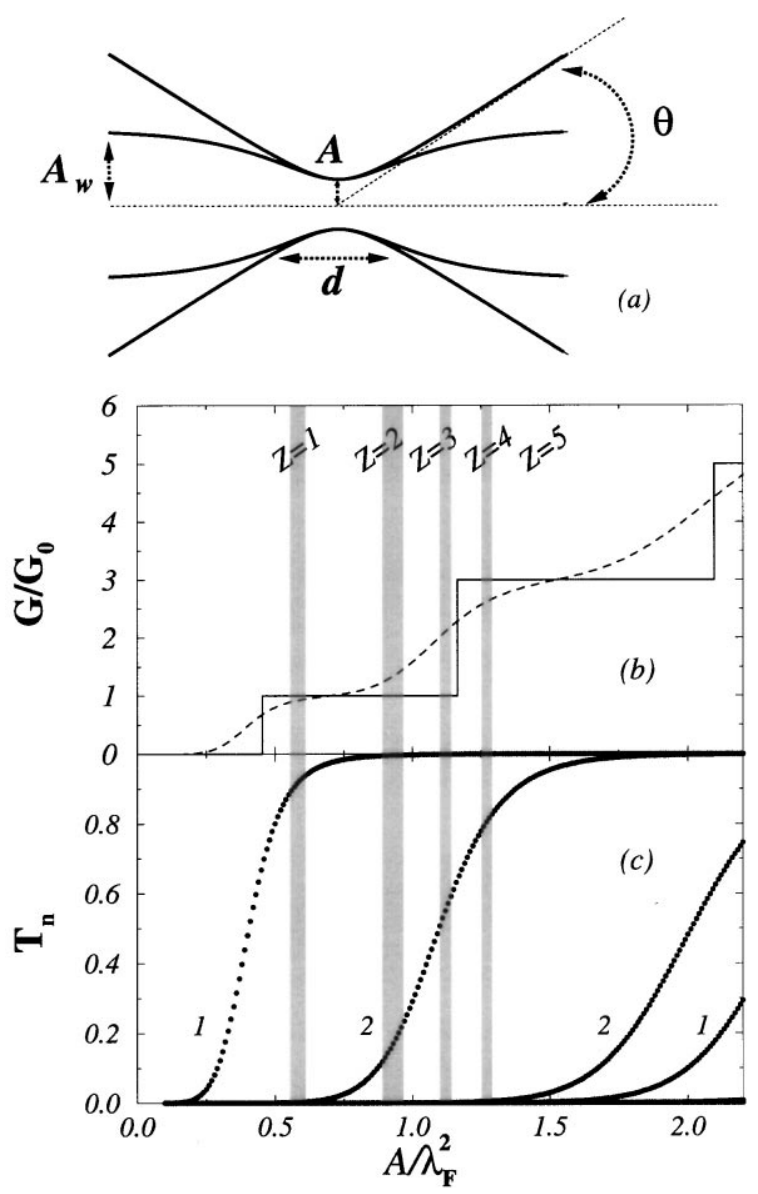

Fig. 1. (a) Schematic view of the contact models. (b) $G / G_{0}$ versus $A / \lambda_{\mathrm{F}}^{2}$ for hyperbolic constrictions, $\theta=0^{\circ}$ (solid line) and $\theta=60^{\circ}$ (dashed line). (c) Transmission probabilities of each conductance eigenchannel for $\theta=60^{\circ}$ (the degeneracy of each channel is also indicated). The shaded regions indicate the effective single-atom cross-section for typical metals with different number $Z$ of valence electrons.

(finite work function) correction [19]. The shaded regions in Fig. 1b indicate the effective atomic cross-sections for typical metals with different numbers $Z$ of valence electrons.

The number of channels $N$ critically depends on the symmetry of the contact. In the case of a single atom contact or an atomic wire, the symmetry is expected to be essentially cylindrical. In this case, the expected values for $N$ are $1,3,5,6, \ldots$ (this is imposed by the essential axial degeneracy $[15,20])$. Notice that the expected values (see 
Fig. 1) are exactly the same as those deduced from the experiments ${ }^{2}$ for $\mathrm{Au}(Z=1, N=1), \mathrm{Al}(Z=3$, $N=3), \mathrm{Pb}(Z=4, N=3)$ and $\mathrm{Nb}(Z=5, N=5)$ single atom contacts [10]. For $Z=1(\mathrm{Au})$ there is only one propagating (open) channel with $G$ close to $G_{0}$ independently of $\theta$. In contrast, for $Z=3$ (A1) both $G$ and $N$ change with $\theta$ : for $\theta \approx 0^{\circ}$ there is only one propagating channel with $G \approx G_{0}$ and, as $\theta$ increases, $G$ increases up to $G \approx 2 G_{0}\left(\theta \approx 60^{\circ}\right)$ with one open channel and two degenerate evanescent (tunneling) modes. The estimated free electron atomic conductances as well as the number of channels follow surprisingly well the results of both 'tight-binding' and 'ab initio' calculations for Al contacts [21-23].

For larger contacts the conductance depends on the interplay between electronic and mechanical properties, and a detailed theoretical description is a very difficult task. A qualitative picture, however, can be obtained from a very simple model system based on the saddle-point-contact model $[24,25]$. In this model the constriction geometry is given by the equipotential surface $V(x, y, z)=E_{\mathrm{F}}$, where $E_{\mathrm{F}}$ is the electron kinetic energy at the narrowest part of the constriction. In our case, we introduce a three-dimensional confining potential that, in units of $E_{\mathrm{F}}$, can be expressed in the form:

$$
V(x, y, z)=\frac{\pi}{\eta} \frac{x^{2}}{A}+\pi \eta \frac{y^{2}}{A}-\tau \tanh ^{2}\left(\frac{z}{d}\right) .
$$

The conduction takes place along the $z$-direction, whereas the electrons are confined in the transverse directions by parabolic potentials. The constriction geometry as sketched in Fig. 1a looks like a smooth wide-narrow-wide geometry with 'elliptical' crosssection. $d$ is the constriction length, $A$ and $A_{\mathrm{w}}$ are the areas of the narrow and wide sections, $\tau=\left(A_{\mathrm{W}} / A-1\right)$ and $\eta$ describes the degree of anisotropy $[24,25]$ of the elliptical cross-section ${ }^{3}$. We may also define in this case an opening angle $\theta$ given by $\tan \theta=\sqrt{A_{\mathrm{W}}-A} / \sqrt{\pi} d$. In the limit

\footnotetext{
${ }^{2}$ In the experiments $[9,10]$, channels with a transmission above $1 \%$ are considered as contributing channels.

${ }^{3}$ In the case of gold contacts, the experimental conductance histograms present clear peaks at $1,2,3, \ldots, G_{0}[5-7]$, reflecting the absence of channel degeneracy. In order to mimic this behavior, we take $\eta=0.67$.
}

$A_{\mathrm{W}} / A \gg 1, T_{n m}$ takes the simple form of a Fermi function:

$T_{n m}=\left[1+\exp \left(\frac{-2 \pi\left(\sqrt{\pi A}-\epsilon_{n m}\right)}{\tan \theta}\right)\right]^{-1}$,

with $\epsilon_{n m}=(n+1 / 2) / \sqrt{\eta}+\sqrt{\eta}(m+1 / 2)$ (all lengths are in units of $\lambda_{\mathrm{F}}$ ).

Let us assume that, for small contacts, the cross-section $A$ follows an almost exponential dependence on the displacement $d$ [26-29]. The qualitative results will not depend on the exact dependence, however. In the inset to Fig. 2 we have plotted $G$ versus the elongation $d$. Except for the last plateau, the quantum nature of transport manifests itself as small oscillations (due to the onset of new propagating channels) superimposed on an otherwise smooth curve. These tiny oscillations are enough to give rise to clear peaks in the histograms: assuming that the actual conductance can take any value along these curves, we obtain the conductance histogram showed in Fig. 2. Despite the simplicity of the model, the histogram obtained is strikingly similar to experimental gold histograms [5-7]. Moreover, the number of channels associated with each conductance value is consistent with that deduced from experiments on $\mathrm{Au}$ contacts [10].

In summary, the conductance and transport channels in atomic scale contacts depend not only

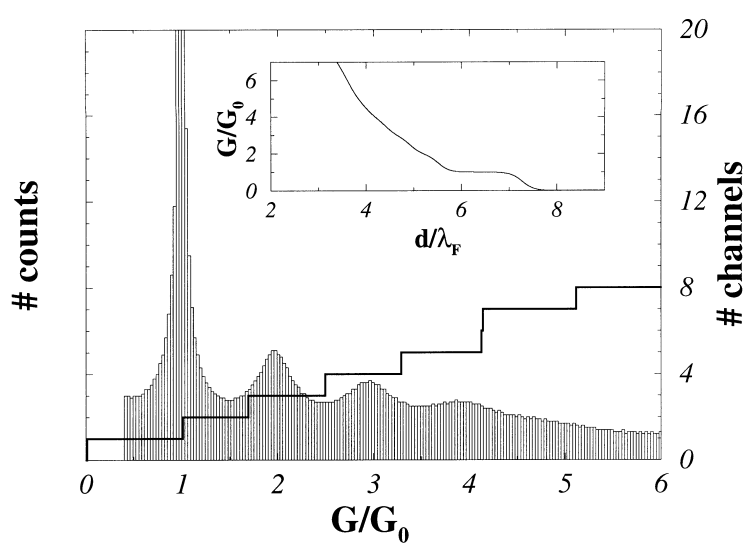

Fig. 2. Conductance histogram obtained from the averaged conductance $G / G_{0}$ versus displacement $d / \lambda_{\mathrm{F}}$ shown in the inset. The stepped line (right axis) indicates the number of channels contributing to each conductance value. 
on the chemical nature of the contact but also on the local geometry. For large opening angles, tunneling of evanescent channels contributes significantly to the conductance leading to the smearing of quantum plateaux and, in general, the histogram peaks at integral multiples of $G_{0}$ are not a signature of conductance quantization of individual contacts. However, the observed peaks can still be associated with the quantum nature of electron transport in metallic contacts.

\section{Acknowledgement}

We acknowledge stimulating discussions with N. Agraït, E. Bascones, J.C. Cuevas, G. GómezSantos, F. Guinea, A. Levy-Yeyati, A. MartínRodero, G. Rubio, P. Serena, J.M. Soler, C.A. Stafford, M. Tsukada, C. Urbina and J. van Ruitenbeek. This work has been supported by the DGICyT through Grant No. PB95-0061. A.J.C. has been supported by DGICyT interchange program (No. IN92-D00830413) through joint agreement between RYMSA (Radiación y Microondas, SA) and the UAM.

\section{References}

[1] P.A. Serena, N. García (Eds.), NanowiresNATO-ASI Ser. E: Appl. Sci. Vol. 340, Kluwer, Dordrecht, 1997.

[2] J.M. van Ruitembeek, in: L.L. Shon, L.P. Kouwenhoven, G. Schön (Eds.), Mesoscopic Electron Transport, Kluwer, Dordrecht, 1997, pp. 549-579.

[3] M. Brandbyge, M.R. Soresen, K.W. Jacobsen, Phys Rev. B 56 (1997) 14956.

[4] C.A. Stafford, D. Baeriswyl, J. Bürki, Phys Rev. Lett. 70 (1997) 2863.

[5] L. Olesen, E. Laegsgaard, I. Stensgaard, F. Besenbacher,
J. Schiotz, P. Stoltze, K.W. Jacobsen, J.K. Norskov, Phys. Rev. Lett. 74 (1995) 2147.

[6] J.M. Krans, J.M. van Ruitenbeek, V.V. Fisun, L.J. Yason, L.J. de Jongh, Nature 375 (1995) 767.

[7] J.L. Costa-Krämer, N. García, P. García-Mochales, P.A. Serena, Surf. Sci. Lett. 342 (1995) L1144.

[8] A.I. Yanson, J.M. van Ruitenbeek, Phys. Rev. Lett. 79 (1998) 2157.

[9] E. Scheer, P. Joyez, D. Esteve, C. Urbina, M.H. Devoret, Phys. Rev. Lett. 78 (1997) 3535.

[10] E. Scheer, N. Agrait, J.C. Cuevas, A. Levy Yeyati, A. Martín-Rodero, G. Rubio-Bollinger, J.M. van Ruitenbeek, C. Urbina, Nature 394 (1998) 154.

[11] E. Bascones, G. Gómez-Santos, J.J. Sáenz, Phys. Rev. B 57 (1998) 2541.

[12] C. Kittel, Introduction to Solid State Physics, 6th ed., Wiley, New York, 1986, Chapter 6.

[13] N.W. Ashcroft, N.D. Mermin, Solid State Physics, W.B. Saunders, San Francisco, CA, 1976, Chapter 2.

[14] A.P. Sutton, Electronic Structure of Materials, Clarendon Press, Oxford, 1993, Chapter 8.

[15] J.A. Torres, J.I. Pascual, J.J. Sáenz, Phys. Rev. B 49 (1994) 16581.

[16] T. Kizuka, K. Yamada, S. Deguchi, M. Naruse, N. Tanaka, Phys. Rev. B 55 (1997) R7398.

[17] H. Ohnishi, Y. Kondo, K. Takayanagi, Nature 395 (1998) 780.

[18] A.I. Yanson, G. Rubio-Bollinger, H.E. van der Brom, N. Agraït, J.M. van Ruitenbeek, Nature 395 (1998) 783.

[19] A. García-Martín, J.A. Torres, J.J. Sáenz, Phys. Rev B 54 (1996) 13448.

[20] E.N. Bogachek, A.M. Zagoskin, I.O. Kulik, Sov. J. Low Temp. Phys. 16 (1990) 796.

[21] N.D. Lang, Phys. Rev. Lett. 79 (1997) 1357.

[22] C.C. Wang, J.L. Mozos, G. Taraschi, J. Wang, H. Guo, Appl. Phys. Lett. 71 (1997) 419.

[23] M. Tsukada, N. Kobayasi, J.L. Brandbyge, Proceedings of the 45th International Field Symposium, Irbid, Jordan 1998.

[24] M. Büttiker, Phys. Rev. B 41 (1990) 7906.

[25] A.G. Scherbakov, E.N. Bogachek, U. Landman, Phys. Rev. B 53 (1996) 4054.

[26] J.A. Torres, J.J. Sáenz, Phys. Rev. Lett. 77 (1996) 2245.

[27] J.A. Torres, J.J. Sáenz, Physica B 218 (1996) 234.

[28] A. Stalder, U. Dürig, Appl. Phys. Lett. 68 (1996) 637.

[29] C. Untiedt, G. Rubio, S. Vieira, N. Agraït, Phys. Rev. B 56 (1997) 2154. 